\title{
Ein neues grundlegendes Werk über die baltischen Lehnwörter
}

SANTERI Junttilla: Tiedon kumuloituminen ja trendit lainasanatutkimuksessa. Kantasuomen balttilaislainojen tutkimushistoria [Die Kumulation des Wissens und die Trends in der Lehnwortforschung. Die Geschichte der Erforschung der baltischen Lehnwörter im Urfinnischen]. Helsinki 2015.

\section{Allgemeines}

Thema der Dissertation von Santeri Junttila sind die alten baltischen Lehnwörter in den ostseefinnischen Sprachen. Es handelt sich um ein von den üblichen etymologischen Dissertationen abweichendes, theoretisches und forschungsgeschichtliches Werk. Sein Gegenstand sind die Vermehrung und Präzisierung des Wissens über Lehnwörter in der Forschungsgeschichte, die Argumente der wissenschaftlichen Gemeinschaft für und wider Lehnetymologien sowie die Identifizierung von in unterschiedlichem $\mathrm{Ma} ß$ als sicher geltenden baltischen Lehnwörtern.

Der hier zu besprechende Band ist eine sorgfältig geschriebene und ansprechend gedruckte Monografie, eine heutzutage rar gewordene
Art der Veröffentlichung von Dissertationen. Die Untersuchung ist strukturell, textuell und stilistisch hochwertig und knüpft an die besten Traditionen der finnischsprachigen Sachprosa an. Statt im Selbstverlag hätte sie in einer renommierten $\mathrm{Pu}$ blikationsreihe erscheinen sollen, sofern noch irgendeine Institution bereit wäre, finnischsprachige Dissertationen zu drucken. Bei der Lektüre des schön gebundenen und typografisch gestalteten Buches mag man darüber nachdenken, ob künftig noch jemand die Zeit und die Bereitschaft aufbringt, ein so sorgfältig ausgearbeitetes finnischsprachiges wissenschaftliches Werk zu verfassen und zu drucken.

Den Anforderungen der heutigen Zeit entsprechend ist das Werk glücklicherweise auch kostenlos im Internet verfügbar, sodass jeder die Möglichkeit hat, sich mit ihm bekannt zu machen.

\section{Fragestellung und Kontext}

Während die indogermanische Komparativistik sich vor allem auf die Rekonstruktion des Wortschatzes der Ursprachen verschiedener Ebenen konzentriert hat, war die 
uralische Komparativistik vornehmlich Lehnwortforschung.

Gerade in der Lehnwortforschung wurde im Bereich der finnisch-ugrischen, insbesondere der ostseefinnischen Sprachen ein methodologischer Fortschritt erreicht, der auch bei der Untersuchung des Wortschatzes anderer Sprachfamilien genutzt werden kann. Dies liegt an der Verknüpfung der uralischen und der indogermanischen historischen Wortschatzforschung. Die Uralistik hat das gesamte gut rekonstruierte Ursprachensystem der Indogermanistik vor allem bei der Untersuchung der Wortschatzschichten der westlichen uralischen Sprachen genutzt. Gleichzeitig bietet sie im Prinzip der indogermanischen Komparativistik ein Wortschatzmaterial, das die verschiedenen Ursprachen der Sprachfamilie zeitlich und örtlich verankert, auch wenn nicht viele Indogermanisten dieses Material gut kennen oder nutzen.

Vor allem Jorma Koivulehto und die an ihn anknüpfende Forschung im 21. Jahrhundert haben historisch alte und lautlich komplizierte, aber dennoch glaubwürdige Lehnetymologien identifiziert. In diesem Sinne ist eine kritische methodologische Betrachtung der Erforschung des uralischen Lehnwortschatzes sehr willkommen, und seine potentielle Bedeutung reicht auch über die Uralistik hinaus.

Der baltische Lehnwortschatz eignet sich gut für eine theoretische Betrachtung der Lehnetymologien, weil er aus der Perspektive der ostseefinnischen nicht der zentralste oder die größte Leidenschaft weckende Untersuchungsgegenstand war. Daher werden in diesem Forschungsbereich die üblichsten Argumentationstypen der Lehnwortforschung relativ offen sichtbar. Andererseits überschneidet sich die Untersuchung der baltischen Entlehnungen mit derjenigen der slawischen Lehnwörter, die in vielerlei Hinsicht komplizierter ist, denn es gab zahlreiche lehngebende Dialekte, von denen offenkundig archaisch waren und zudem in vielerlei Hinsicht den baltischen Sprachen glichen. Es ist denn auch offensichtlich, dass die Grenzziehung zwischen dem baltischen und dem slawischen Wortschatz in der Forschung noch nicht völlig zufriedenstellend durchgeführt wurde, soweit sie überhaupt bei jedem einzelnen Wort möglich ist.

Eine in gewisser Weise entsprechende finnougristische Dissertation wurde 1983 vorgelegt; damals bot die Untersuchung Itämerensuomen vanhimmasta sanastosta ja sen tutkimisesta [Über den ältesten Wortschatz des Ostseefinnischen und seine Erforschung] von 
Kaisa Häkkinen eine vergleichbare begriffliche Klärung für die Erforschung des alten Wortschatzes der uralischen Sprachen. Die Verfasserin zeigte anschaulich auf, dass die zur Unterstützung vieler Ursprachenstufen (z. B. FinnischWolgaisch, Finnisch-Permisch) angeführte komparative Evidenz tatsächlich recht geringfügig war.

Wie in der damaligen Dissertation von Häkkinen wird auch in Junttilas Werk gut systematisiert, was in der Forschungsgeschichte bisher getan wurde und welche etymologischen Argumente verwendet wurden, um die Forschung voranzubringen, doch es werden kaum neue Etymologien für einzelne Wörter vorgelegt. Es sei jedoch erwähnt, dass diese bei Junttila häufiger sind als bei Häkkinen. Er verwirft nämlich mit ausführlicher Begründung eine ganze Reihe postulierter baltischer Lehnwörter (u.a. die Wörter porsas, taivas, kaikki und aitta), präsentiert eine Reihe neu begründeter (ruskea, rauta, hauki u. a.) und sogar einige ganz neue Etymologien (u.a. für die Wörter kylki und leuka, dazu unten mehr).

Neue etymologische Entdeckungen dieser Art sind zwar interessant, aber für die Gesamtheit der vorliegenden Arbeit dennoch nebensächlich. Der Schwerpunkt liegt auf der Analyse der Methoden der etymologischen Forschung. In seiner derzeitigen Form ist Junttilas Werk gewissermaßen eine sorgfältig abgefasste Einleitung zu einer noch nicht erschienenen Abhandlung über die baltischen Lehnwörter in den ostseefinnischen Sprachen. Der Verfasser geht die Geschichte der Erforschung der baltischen Entlehnungen mit gerade$\mathrm{zu}$ pedantischer Genauigkeit und gruppiert die verschiedenen Typen von lautlichen und semantischen Argumenten, die in der Forschung vorgebracht wurden, untersucht aber letztlich weniger die baltischen Entlehnungen selbst, sondern vor allem das, was über sie gesagt wurde. Es fehlt eine eigene systematische und kritische Beurteilung der baltischen Etymologien, obwohl außer Zweifel steht, dass der Verfasser fähig gewesen wäre, auch diese Aufgabe zu meistern.

Es handelt sich also hauptsächlich um eine Untersuchung der Meta-Ebene. Dagegen ist an sich nichts einzuwenden, doch die vorliegende Arbeit gibt allen Anlass, eine Fortsetzung zu erwarten. Aufgrund des Materials von Junttila wäre es leicht, z. B. ein Wörterbuch der baltischen Lehnwörter in der Art des LÄGLW zu erstellen, was der Verfasser auch ankündigt. TatsächIich findet sich der größte Teil der relevanten Informationen bereits in dem vorliegenden Band, 
es fehlt nur noch ihre Systematisierung in Wörterbucheinträgen und vor allem ein verstärkter kritischer Eigenbeitrag des Verfassers.

\section{Material und Methoden}

Das Material von Junttilas Werk ist überaus umfassend; es besteht im Prinzip aus allen Untersuchungen, in denen ein altes baltisches Lehnwort in den ostseefinnischen Sprachen erwähnt wird. Junttila zufolge enthält das Gesamtmaterial mehr als 27000 Erwähnungen oder Behauptungen über die Herkunft von Wörtern. Vor allem hinsichtlich der in den baltischen Sprachen und in Estland publizierten Untersuchungen ergänzt Junttila das Bild, das die finnischen Wissenschaftler von der Erweiterung des etymologischen Wissens haben dürften, in erheblichem Umfang. In Bezug auf die in Russland erschienene Forschung kann seine Untersuchung dagegen nicht als ganz vollständig gelten (s. unten).

Junttilas Arbeit dürfte die erste etymologische Monografie sein, in der das Material des etymologischen Wörterbuchs des Estnischen (EES) in seiner Gesamtheit berücksichtigt werden konnte. Sie markiert insofern den Übergang von der auf das Finnische konzentrierten Etymologisierung der ostseefinnischen Sprachen $\mathrm{zu}$ einer genaueren Berücksichtigung der lexikalischen Evidenz des gesamten Ostseefinnischen.

Die Belesenheit des Verfassers sucht selbst unter den in aller Regel sehr belesenen Finnougristen ihresgleichen und ist sicher größer als die der meisten von ihm beurteilten Wissenschaftler. Dennoch habe ich einige Lücken im Material gefunden. Z.B. wird das auf die Ortsnamen gegründete ablehnende Urteil von Matveev (2002) über die baltische Etymologie des Wortes järvi nicht erwähnt, ebensowenig der diesbezügliche Hinweis des Rezensenten (2006); auch meine Erwähnungen u.a. zu den Wörtern palva und palvoa sowie einigen anderen (Saarikivi 2009) habe ich nicht gefunden. Besonders die Erwähnung des Wortes järvi wäre notwendig gewesen, denn darin wird eine von anderen Quellen substantiell abweichende Auffassung von der ursprünglichen Form

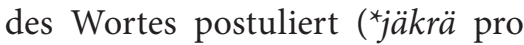
*järwä).

Im Hinblick auf die Gesamtheit der Untersuchung haben solche Lücken natürlich keine Bedeutung, doch sie zeigen, wie schwierig es ist, selbst einen so kleinen Komplex innerhalb der ostseefinnischen Etymologie zu beherrschen, wie ihn die Literatur zu allen baltischen Lehnwörtern darstellt. Junttila weist denn auch mehrfach darauf 
hin, dass es in der Geschichte der Erforschung der baltischen Lehnwörter wiederholt vorkommt, dass dieselbe Etymologie unabhängig voneinander mehrmals vorgestellt wird. Dies zeigt, wie wichtig es wäre, die alte Forschungsliteratur zu digitalisieren, um sie heutigen Wissenschaftlern zugänglich $\mathrm{zu}$ machen, und Datenbanken zu schaffen, in denen die Erwähnungen in der alten Forschung durch Suchmaschinen zu finden sind.

Der Verfasser hat alle Herkunftsangaben, die das Primärmaterial seiner Untersuchung bilden, sorgfältig tabelliert und gezählt. Dieses Material wird auf vielerlei verschiedene Weise statistisch behandelt, u. a. zeitlich und nach den Argumentationstypen, und die Ergebnisse werden in Kurven und Diagrammen präsentiert, die den zeitlichen Wandel sichtbar machen. Das umfangreiche Tabellenmaterial, das der Verfasser erstellt hat, ist auf derselben Webseite der Universität Helsinki verfügbar wie die Dissertation selbst. Es ist zu hoffen, dass eine solche öffentliche Verbreitung von Untersuchungsmaterial sich zum Standard des Faches entwickelt.

Da der Verfasser nach eigenen Angaben den größten Teil des alten Untersuchungsmaterials gescannt hat, fragt man sich, ob nicht auch dieses Material anderen Forschern z. B. auf einer Webseite zur Verfügung gestellt werden sollte. Zumindest könnte man den Teil der alten Literatur wiederveröffentlichen, bei dem keine urheberrechtlichen Probleme vorliegen.

\section{Urfinnisch, Urbaltisch und die Identifizierung des mit diesen ver- knüpften Wortschatzes}

Junttila schreibt, das Thema seiner Arbeit seien die urfinnischen baltischen Entlehnungen im Ostseefinnischen. Das urfinnische Stadium wird ausschließlich lautgeschichtlich definiert, sodass als urfinnisch alle Entlehnungen gelten, die die baltoslawische Satemisation, nicht aber die Lautwandel der einzelnen ostseefinnischen Sprachen durchlaufen haben.

Da das Urfinnische sich jedoch in viele historische Stadien gliedert und von seiner lautlichen Entwicklung her komplex ist, kann man fragen, ob alle in dem vorliegenden Band behandelten Wörter wirklich urfinnisch sind. Aus vielen Kontexten kennt man Situationen, in denen ein scheinbar alt wirkender Wortschatz mit Sicherheit recht jung ist (Aikio 2007) oder in denen in derselben Lehnwortschicht parallel mehrere Substitutionsmodelle begegnen, die sich dennoch altersmäßig nicht voneinander unterscheiden (z.B. Grönholm 
1988, in Bezug auf die schwedischen Lehnwörter im Dialekt von Turku, oder Must 2000, in Bezug auf die slawischen Entlehnungen in den estnischen Dialekten). Aus Gründen dieser Art ist die auf rein lautlichen Kriterien basierende Behauptung, dieses oder jenes Wort sei urfinnisch, häufig ein wenig befremdlich.

Es wäre ratsam, daneben auch mit der Verbreitung zu argumentieren, besonders, da die Lautgeschichte des Urfinnischen immer noch nicht so deutlich dargestellt werden kann, dass die Phonotaxe der urfinnischen Wörter in allen Einzelheiten bekannt wäre. Zumindest können z. B. die mit Affrikate anlautenden Wörter, die z.B. im Karelischen begegnen (čiitalo 'Rückstand auf dem Grund von geschmolzenem Fett', S. 123), nicht urfinnisch sein. Desgleichen verwirft Junttila selbst (S. 251) die baltische Herkunft u.a. der Wörter aitta, kaikki, kiittää usw., weil ihre Phonotaxe mit den frühen Stadien des Urfinnischen unvereinbar ist, und äußert auch selbst die Vermutung, dass einige der von ihm behandelten baltischen Entlehnungen relativ jungen Datums sind, selbst dann, wenn ihre Verbreitung bis in das östliche Ostseefinnische reicht (aatkela, puusniekka, S. 239-242).

Ein entsprechendes Problem stellt sich auch bei der Definition der baltischen Herkunft der Wörter, ganz besonders, wenn ein Wort aufgrund seiner im Slawischen erhaltenen Entsprechung als baltische Entlehnung ins Urfinnische identifiziert wird.

Heute neigen wohl die meisten Sprachhistoriker das Urslawische im Wesentlichen für eine Weiterentwicklung des Urbaltischen $\mathrm{zu}$ halten. Dies eröffnet im Prinzip die Möglichkeit, im Slawischen attestierte, aber im heutigen Baltischen fehlende, von ihrer lautlichen $\mathrm{Ge}$ stalt her archaische ostseefinnische Wörter als baltische Entlehnungen darzustellen. Beispielsweise postuliert Junttila, dass rauta und leuka baltische Wörter sein könnten, deren Entsprechungen im Slawischen erhalten geblieben sind. In diesem Zusammenhang übergeht er jedoch den in der Forschung schon seit langem bekannten Umstand, dass in der Ostseeregion offenbar auch aus den slawischen Sprachen selbst Wörter entlehnt wurden, in denen die alten Diphthonge vertreten sind. U. a. heißt der Fluss Laukaanjoki auf Russisch Luga, und schon in der frühen Forschung galt dies als Argument dafür, dass die Diphthonge der ersten Silbe noch nicht geschwunden waren, als die Vorformen des Slawischen an die Ufer der Ostsee gelangten.

Aber wie wäre im Licht dieses Wissens die „baltische Herkunft“ 


\section{z. B. des Wortes rauta zu beurteilen?} Es begegnet in der dem Ostseefinnischen entsprechenden Bedeutung 'Erz' vornehmlich im Russischen (und in anderen slawischen Sprachen, vgl. russ. ruda $<{ }^{*}$ rauda). Im heutigen Baltischen hat das Wort vor allem die Bedeutung 'rote Farbe', wie auch im Germanischen, wo allerdings die Bedeutung 'Sumpferz' bekannt ist.

Entsprechend gab es im Slawischen auch Dialekte, in denen die das Slawische vom Baltischen unterscheidende sog. 2. Palatalisierung, die das $k$ vor einem Vordervokal in $c$ verwandelt, nicht stattgefunden hat. Über die Dialekte des Slawischen in den Gebieten von Novgorod und Pihkova, die baltische Elemente bewahrt haben, gibt es relativ viel Literatur, und es wäre nicht verwunderlich, wenn viele als baltisch angesehene Entlehnungen tatsächlich aus diesen Dialekten stammten.

Bei der Prüfung der Herkunftssprache von Wörtern wäre es angebracht, auch die Möglichkeit zu berücksichtigen, dass ein Teil des als baltisch betrachteten Wortschatzes in Wahrheit sowohl im Ostseefinnischen als auch im Baltischen aus einer dritten Quelle stammt. Deshalb sollte auch der indogermanische Hintergrund eines baltischen Wortes zu einem gewissen Grad im Auge behalten werden. Im
Ostseegebiet scheint es einen recht großen Wortschatz zu geben, dessen Verbreitung auf das Ostseefinnische, Baltische / Baltoslawische und Germanische beschränkt ist. Unter den traditionell als germanisch oder baltisch betrachteten Lehnwörtern sind nämlich recht viele, deren indogermanische Äquivalentreihen beschränkt sind und die eher als areale Innovationen denn als indogermanischer Erbwortschatz erscheinen.

\section{Die Terminologie der Etymologie}

Junttila hat die bei der Untersuchung von Etymologien verwendeten Argumente der Form, der Bedeutungsentsprechung, der Verbreitung und andere Standardargumente sehr eifrig und im Prinzip sinnvoll klassifiziert. Viele Teile dieser Klassifizierung dürften in der künftigen Forschung gut $\mathrm{zu}$ verwenden sein, u.a. die Unterscheidung zwischen Gleichsetzung (Fi. rinnastus) und Herkunftsbehauptung (Fi. alkuperäväite) (Junttila zufolge lautet z. B. in der Etymologie „fi. helle $\leftarrow$ balt., vgl. lt. šiltis "Wärme" die Herkunftsbehauptung „fi. helle $\leftarrow$ balt." und die Gleichsetzung „fi. helle 1t. šiltis 'Wärme'“, S. 53). Ein Hinweis auf die Anwendungsmöglichkeiten der von Junttila geschaffenen neuen Terminologie ist u.a. die Tatsache, 
dass der Rezensent die Trennung von Gleichsetzung und Herkunftsbehauptung bereits in anderem Kontext genutzt hat (s. Saarikivi, in diesem Heft).

Das Werk enthält jedoch auch eine nicht geringe Menge an neuer Terminologie, deren Anwendbarkeit sich erst noch erweisen muss. Zwar basieren alle von Junttila beschriebenen Argumentationsweisen auf der bisherigen etymologischen Literatur, doch dürfte in der Forschung bisher z. B. nicht vom „Verbreitungslückenargument" (Fi. levikkiaukkoargumentti) die Rede gewesen sein. Mit diesem Wort wird auf einen Wortschatz verwiesen, der technisch gesehen baltischer oder baltoslawischer Herkunft sein könnte, der jedoch in hinsichtlich der Lehnkontakte zentralen Quellen fehlt. Auch dürfte man bisher nicht vom „Entlehntheitsargument" (Fi. lainautuneisuusargumentti) gesprochen haben - es bezieht sich auf einen Wortschatz, von dem vermutet wird, er sei baltisch, der aber in den baltischen Sprachen selbst eine offenbar junge Entlehnung ist.

Junttilas Klassifizierung und Benennung der Argumente leidet unter einem gewissen Solipsismus. Obwohl es sich um eine wissenschaftsgeschichtliche Untersuchung handelt, wurden die Argumente oder Gegenargumente nicht mit wissenschaftsphilosophischen Methoden untersucht. Es liegt auf der Hand, dass sich unter ihnen, wie auch in jeder anderen Forschung, in reichem Maß z. B. Berufung auf Autoritäten, Verwendung oder Nichtverwendung von Analogien, ad hoc-Lautbeziehungen u. ä. finden.

Eine deutlichere Verknüpfung der Terminologie mit der Tradition der Wissenschaftsphilosophie hätte möglicherweise auch die Aufmerksamkeit eines nicht mit der Etymologie vertrauten, aber an der Geschichte der humanistischen Forschung interessierten Publikums geweckt. So aber besteht die Gefahr, dass ein Teil der an sich gut begründeten Terminologie sich nie über das vorliegende Werk hinaus verbreitet, allein schon deshalb, weil die etymologische Forschung von ihren Methoden her international ist und zum größten Teil nicht auf Finnisch publiziert wird.

\section{Analyse des Materials}

Den größten Teil des Bandes nimmt die Analyse der Argumente für die Zuverlässigkeit der in der Forschung vorgelegten Lehnetymologien und ihrer Begründungen in Anspruch. Als erstes führt Junttila an, welche baltischen Lehnetymologien vorgeschlagen und wie sie aufgenommen wurden. Daraus 
ergibt sich zunächst eine scheinbare „Konsensmethode“, bei der als die sichersten baltischen Lehnwörter diejenigen gelten, die in der Forschung mehr Unterstützung als Kritik erhalten haben. Junttila klassifiziert die Etymologien seines Materials als „gutgeheißen“, „umstritten“, „kritisiert“, „verworfen“ usw.

Das ist natürlich eine mögliche Methode, um den gegenwärtigen Stand der Wissenschaft darzustellen, doch in Bezug auf die Glaubwürdigkeit wissenschaftlicher Behauptungen handelt es sich dennoch um die Aufhäufung wissenschaftlicher Erkenntnisse ex autoritate, was einen der gröbsten Argumentationsfehler darstellt. Bei der wissenschaftlichen Glaubwürdigkeit darf es nie um irgendeine Art von demokratischer Abstimmung gehen. Eine äußerst unpopuläre Erklärung kann im Prinzip die einzig richtige und eine extrem populäre falsch sein. Anstelle der Kumulation von Wissen kennt die Forschungsgeschichte zahlreiche Fälle, in denen die nicht hinterfragte Autorität eines Wissenschaftlers oder einer Methode die Forschung paradigmatisch lenkt, so dass dieselbe Wahrheit von einer Untersuchung zur anderen wiederholt wird, ohne dass man sie je in Frage stellt.
Zum Glück beschreibt der Verfasser im letzten Teil seiner Untersuchung auch die mit den Herkunftsbehauptungen des baltischen Wortschatzes verknüpften etymologischen Argumente. In diesem Zusammenhang verwirft er einige bisher gutgeheißene Etymologien und liefert für verworfene eine neue Begründung. Teilweise unklar bleibt jedoch, wie systematisch diese Analyse ist. Die Systematik erstreckt sich sicher auf alle Argumenttypen, aber erstreckt sie sich auch auf alle Wörter? Es entsteht der Eindruck, dass die Wörter von der Forschungsgeschichte ausgehend beschrieben werden, um einzelne Argumentationstypen $\mathrm{zu}$ illustrieren, und in diesem $\mathrm{Zu}$ sammenhang wird die frühere Forschung auch kritisiert. Andererseits bleibt ein großer Teil der untersuchten Wörter ohne Erwähnung, so dass der Leser nicht erfährt, wie der Verfasser die Glaubwürdigkeit ihrer Etymologie beurteilt.

Soweit der Verfasser seine eigene Analyse vorlegt, ist sie methodologisch folgerichtig und hochwertig. Nur in einigen Punkten hätte die Darlegung der slawischen Dialektologie oder eines größeren indogermanischen Rahmens eventuell etwas wesentlich Neues zu den behandelten Etymologien beitragen können. 


\section{Zum Schluss}

Santeri Junttilas Doktorarbeit steht trotz einzelner Mängel auf bedeutend höherem Niveau als durchschnittliche Dissertationen. Sie enthält das zentrale Material für ein hoffentlich erscheinendes Kompendium der baltischen Lehnwörter in den ostseefinnischen Sprachen. Zugleich steckt sie den Weg $\mathrm{ab}$ für eine methodisch präzisere Untersuchung von Lehnetymologien vor allem im ostseefinnischen Kontext, prinzipiell aber auch darüber hinaus. Der Verfasser zeigt, wie als baltisch anzusehende Entlehnungen u.a. mit semantischen und lautlichen Kriterien falsifiziert werden können, wie andererseits aber weiterhin zahlreiche Entlehnungen zu finden sind, wenn man die Betrachtung in Richtung des Slawischen erweitert.

Es ist $\mathrm{zu}$ hoffen, dass Junttila seine etymologische Arbeit im Bereich der Uralistik fortsetzt, sich zugleich aber künftig bemüht, seine Erkenntnisse in größerem Maß mit der internationalen Linguistengemeinschaft zu teilen.

Janne Saarikivi

\section{Literatur}

Aikio, Ante 2007: Etymological nativization of loanwords. A case study of Finnish and Saami. - Ida Toivonen \& Diane Nelson (eds): Saami linguistics. John Benjamins. Amsterdam \& Philadelphia. 17-52.

EES = Iris Metsamägi \& Meeli Sedrik \& Sven-Erik Soosaar 2012: Eesti etümoloogiasõnaraamat. Eesti keele sihtasutus. Tallinn.

HäkкInen, KaISA 1983: Suomen kielen vanhimmasta sanastosta ja sen tutkimisesta. Suomalais-ugrilaisten kielten etymologisen tutkimuksen perusteita ja metodiikkaa. Turun yliopiston suomalaisen ja yleisen kielitieteen laitoksen julkaisuja 17. Turun yliopisto. Turku.

LÄGLW = Lexikon der älteren Germanischen Lehnwörter der ostseefinnischen Sprachen. Begründet von A. D. Kylstra, fortgeführt von Sirkka-Liisa Hahmo \& Tette Hofstra \& Osmo Nikkilä. Rodopi. Amsterdam \& Atlanta, GA.

Matveev, A. K. 2002 = Матвеев, А. К.: Субтотратные тимнонимь $и$ происхождения названия озер в финских языках. Финно-угорское наследие в русском языке. Сборник научных трудов. Издательство Уральского университета. Екатеринбург.

SAARIKIVI, JANNE 2009: Itämerensuomalais-slaavilaisten kontaktien tutkimuksen nykytilasta. - Ylikoski, Jussi (ed.): The Quaisquicentennial of the Finno-Ugrian Society. Mémoires de la Société Finno-Ougrienne 258. Société Finno-Ougrienne. Helsinki. 109-16o.

- 2016: Das Sumerische ist weiterhin eine isolierte Sprache. (In diesem Band.) 